\title{
P36 Leukocyte Telomere Length is Inversely Associated with Wave Reflection in 566 Normotensive and Never-treated Hypertensive Subjects
}

\author{
Ilkka Pörsti ${ }^{1,2, *}$, Milja Honkonen ${ }^{1}$, Kati Vääräniemi ${ }^{1}$, Outi Saijonmaa ${ }^{3}$, Antti Tikkakoski ${ }^{1}$, Jenni Koskela ${ }^{1,2}$, \\ Arttu Eräranta ${ }^{1}$, Mika Kähönen ${ }^{1}$, Jukka Mustonen ${ }^{1}$, Frej Fyhrquist ${ }^{3}$ \\ ${ }^{1}$ Faculty of Medicine and Health Technology, Tampere University, Tampere, Finland \\ ${ }^{2}$ Clinic of Internal Medicine, Tampere University Hospital, Tampere, Finland \\ ${ }^{3}$ Minerva Institute for Medical Research, Biomedicum U2 Helsinki, 00290 Helsinki, Finland
}

\section{ABSTRACT}

Background: Telomeres are short segments in chromosome ends, the length of which is reduced during cell life-cycles. We examined the association of leukocyte telomere length and short telomere proportion with hemodynamic variables.

Methods: Altogether 566 subjects (279 women and 287 men) without cardiovascular disease and medications with direct cardiovascular influences were subjected supine recordings for 5 minutes. Haemodynamics were captured using continuous tonometric pulse wave analysis and whole-body impedance cardiography. The analyses were adjusted for age, body mass index (BMI), alcohol use, smoking, plasma chemistry, and estimated glomerular filtration rate (eGFR).

Results: In univariate analyses, leukocyte telomere length correlated with age, BMI, eGFR, aortic blood pressure, augmentation index, pulse wave velocity, and systemic vascular resistance $(p<0.05$ for all). Short telomere proportion correlated with age, BMI, eGFR, aortic systolic blood pressure, augmentation index, and pulse wave velocity ( $p<0.05$ for all). In linear regression analyses of all hemodynamic variables, leukocyte telomere length was only an independent explanatory factor for augmentation index (Beta $-0.006, p=0.032$ ), while short telomere proportion was not an explanatory factor for any of the hemodynamic variables, in contrast to age, BMI and several cardiovascular risk factors.

Conclusion: Augmentation index was predominantly related with chronological aging, but also with leukocyte telomere length, suggesting that this variable of central wave reflection is a moderate marker of vascular biological aging.

(c) 2019 Association for Research into Arterial Structure and Physiology. Publishing services by Atlantis Press International B.V. This is an open access article distributed under the CC BY-NC 4.0 license (http://creativecommons.org/licenses/by-nc/4.0/). 